\title{
YM155, specific survivin inhibitor, can enhance artesunate-induced cytotoxicity in HCT116 colon cancer cells
}

\author{
Eui Tae Kim, Dong-Guk Park \\ Department of Surgery, Dankook University College of Medicine, Cheonan, Korea
}

\begin{abstract}
Purpose: A water-soluble variant of the artemisinin called artesunate, approved as an antimalarial agent, can induce cell death on various cancer cell types. We studied the mechanism of cell death of artesunate on HCT116 colorectal cancer cells.

Methods: We treated HCT116 colon cancer cells with artesunate, holo-transferrin, deferoxamine mesylate, ferrostatin, necrostatin-1, and YM155. We observed the growth inhibition of artesunate on HCT116 colon cancer cells by morphologic findings. Inhibition of cell growth was assessed by MTT (3-(4,5-dimethylthiazol-2yl)-2,5-diphenyltetrazolium bromide) assay and long-term growth inhibition by colony-forming assay. Apoptosis was investigated by flow cytometry and Western blot analysis.

Results: Artesunate inhibited the proliferation of HCT116 colon cancer cells effectively. Co-treatment with YM155, a specific survivin inhibitor, enhanced the artesunate-induced cell death. Co-treatment with the iron-chelating agent deferoxamine rescued artesunate induced cell death and increased long-term cell survival and proliferation.

Conclusion: In this study, we demonstrated that artesunate-induced cytotoxicity in HCT116 colon cancer cells by suppressing the expression of survivin and partially by ferroptosis. Our findings suggest that the co-treatment artesunate with YM155 can induce more potent cell death on HCT116 colon cancer cells and shows new insight for the treatment of colorectal cancer patients.
\end{abstract}

Keywords: YM155, Artesunate, Survivin, Necroptosis, Ferroptosis

\section{INTRODUCTION}

Colorectal cancers (CRCs) are the third most common cancers in males and females and the fourth leading cause of death from cancers in Korea in 2014. The incidence rate of colorectal cancers in 2014 was about $12.4 \%$ of all cancers, though it has begun to decrease in both sexes in Korea [1]. The main treatment modalities for colorectal cancers are surgery, radiotherapy, and chemotherapy.

Received: Sep 26, 2020 Revised: Sep 26, 2020 Accepted: Nov 18, 2020 Correspondence to: Dong-Guk Park

Department of Surgery, Dankook University College of Medicine,

119 Dandae-ro, Dongnam-gu, Cheonan 31116, Korea

Tel: +82-41-550-3931, Fax: +82-41-565-6167

E-mail: dkpark@dankook.ac.kr

ORCID: Eui Tae Kim (https://orcid.org/0000-0002-3097-4055), Dong-Guk Park (https://orcid.org/0000-0002-9894-9104)

Chemotherapy is frequently used as the main regimen in most advanced colorectal cancers. However, the development of chemo-resistance and severe side effects present major causes of failure in chemotherapy [2]. Therefore, many researchers have been searching for more effective and less toxic chemosensitizing agents.

Artesunate (ART), a water-soluble derivative of the artemisinin isolated from the Chinese herb Artemisia annua L., is an effective antimalarial agent [3]. Artemisinin contains an endoperoxide moiety that reacts with iron to produce toxic reactive oxygen species (ROS). This lethal ROS production exerts fatal toxicity to the malaria parasites [4].

Cancer cells contain substantial free iron, resulting from their higher rate of iron uptake via transferrin receptors compared with normal cells [5]. This represents artemisinin and its derivatives, such as ART, having toxicity to cancer cells. Many clinical studies on the tolerance and safety of ART as an antimalarial drug have been well documented. Artemisinin and ART have been shown to induce cell death in various cancer cells, including colon, breast, ovarian, gastric, pancreatic, and lung cancer cells [6-10]. Results from in vivo and animal models indicate the potential therapeutic 
activity for cancer treatment in Kaposi's sarcoma, pancreatic cancer, hepatoma, uveal melanoma, and colon cancer [11-17]. Currently, ART is in clinical studies for solid tumors, breast cancer, colorectal cancer, and hepatocellular carcinoma treatment (https:// clinicaltrials.gov). A recent randomized double-blind placebo-controlled study of oral ART therapy for CRC suggested that ART has anti-proliferation properties in CRC and is well tolerated [16].

ART-mediated cytotoxicity is dependent on increased ROS generation and the presence of iron (ferroptosis) $[18,19]$, and by a different mode of programmed cell death, including apoptosis by suppressing survivin expression, enhancing programmed necrosis (necroptosis) [20]. ART may inhibit cancer cell proliferation through a diverse mechanism in different cell types.

Survivin is highly expressed in human cancers, including colorectal cancers, but not detectable in nonproliferating normal adult tissues. The role of survivin in the pathogenesis of cancer is inhibition of apoptosis, regulation of the mitotic spindle checkpoint, promotion of angiogenesis, and chemoresistance [21]. High survivin level is related to poor prognosis and resistance to therapy, radiation, and chemotherapeutic agents [22]. Thus, survivin is a promising target for the sensitization of cancer cells to radiation and chemotherapeutic agents [23]. However, molecular mechanisms of ART-induced cell death in CRC are poorly understood.

In the present study, we investigated the antitumor effect of ART on colon cancer cells. Our data suggest that ART has cytotoxicity in CRC cells by suppressing survivin expression and partially by iron-dependent cell death. ART has potential as a drug candidate for CRC treatment, especially when used in synergy with YM155, a specific survivin inhibitor.

\section{METHODS}

\section{Reagents}

HCT116 human colon cancer cells were obtained from the Korean Cell Bank (Seoul, Korea). Cell culture reagents, penicillin, streptomycin, RPMI 1640, fetal bovine serum (FBS), Hoechst 33258, YM155, crystal violet, ART, holo-transferrin, necrostatin-1, ferrostatin, and deferoxamine mesylate (DFO) were purchased from Sigma (St. Louis, MO, USA). Antibodies for survivin, Bcl-2, caspase-3, actin, and horseradish peroxide (HRP)-conjugated secondary antibody was purchased from Santa Cruz Biotechnology (Santa Cruz, CA, USA), and MTT kit for cell viability assay from Promega (Fitchburg, WI, USA).

\section{Cell culture and drug treatments}

HCT116 colon cancer cells were cultured in RPMI 1640 medium supplemented with $10 \% \mathrm{FBS}, 100 \mathrm{U} / \mathrm{mL}$ penicillin and $100 \mu \mathrm{g} / \mathrm{mL}$ streptomycin in a humidified incubator at $37^{\circ} \mathrm{C}$ and $5 \% \mathrm{CO}_{2}$. Treatments of HCT116 cell lines with ART $(150 \mu \mathrm{M})$, holo-transferrin $(20 \mu \mathrm{g} / \mathrm{mL})$, DFO $(0.1 \mathrm{mM})$, ferrostatin $(20 \mu \mathrm{M})$, Trolox $(0.5$ $\mathrm{mM})$, and necrostatin-1 $(20 \mu \mathrm{M})$ were performed in glucose-containing Hank's balanced salt solution (in mM: $1.3 \mathrm{CaCl}_{2}, 0.5$ $\mathrm{MgCl}_{2}, 0.4 \mathrm{MgSO}_{4}, 5.3 \mathrm{KCl}, 0.4 \mathrm{KH}_{2} \mathrm{PO}_{4}, 4.2 \mathrm{NaHCO}_{3}, 137.9$ $\mathrm{NaCl}, 0.3 \mathrm{Na}^{2} \mathrm{HPO}^{4}, 5.6 \mathrm{D}$-glucose).

\section{Cell viability assay}

A 3-(4,5-dimethylthiazol-2yl)-2,5-diphenyltetrazolium bromide (MTT) assay was used to analyze the viability of HCT116 colon cancer cells after treatment with various agents. Briefly, healthy growing HCT116 cells were detached by trypsin-ethylenediaminetetraacetic acid (EDTA) treatment and $2.5 \times 10^{4}$ cells per ml were resuspended in RPMI containing 10\% FBS, which was then seeded into 96-well culture plates with six duplicates. After 24 hours, the culture was maintained for an additional 48 hours in the absence (control) or presence of ART, holo-transferrin, DFO, Trolox, necrostatin-1, ferrostatin, and YM155 as described in the figure legends. After 48 hours after treatment of reagents, $20 \mu \mathrm{L}$ of $5 \mathrm{mg} / \mathrm{mL}$ stock of MTT was added to each well. After 1 hour incubation, culture media was removed and $0.1 \mathrm{~mL}$ of dimethyl sulfoxide was added to each well to dissolve the formazan crystals. The intensity of color was measured at $570 \mathrm{~nm}$. All values were normalized to control wells.

\section{Colony formation assay}

HCT116 colon cancer cells were subjected to ART with or without DFO. After 24 hours, 300 surviving cells were re-seeded into new 6-well plates and grown in full medium to allow the formation of a colony for a colony-forming assay. After 14 days of re-seeding, the colonies were stained with $1 \%(\mathrm{w} / \mathrm{v})$ crystal violet in $25 \%$ methanol for 20 minutes. Images were acquired using an EOS 60D DSLR camera (Canon, Tokyo, Japan).

\section{Apoptosis and morphologic assay}

HCT116 cells were cultured without or with 100 and $150 \mu \mathrm{M}$ concentration of ART for 48 hours. Morphology of cells was observed in phase-contrast microscopy. Additionally, cells were stained with Hoechst 33,258 and observed in a fluorescent microscope (IX71; Olympus Co., Tokyo, Japan).

Adherent cells $\left(1 \times 10^{6} / \mathrm{mL}\right)$ were collected by trypsinization after treatment of HCT116 cells with or without ART $(60 \mu \mathrm{M})$ for 48 hours, washed twice with PBS, and resuspended in a staining solution containing $1 \mu \mathrm{g} / \mathrm{mL}$ propidium iodide. FACS analysis was performed with the FACScalibur apparatus (Becton Dickinson, 
Franklin Lakes, NJ, USA) and quantification was performed using CELL-Quest software (Becton Dickinson).

\section{Western blot analysis}

The cells were lysed in a lysis buffer $(50 \mathrm{mM}$ Tris, $100 \mathrm{mM} \mathrm{NaCl}$, $2.5 \mathrm{mM}$ EDTA, 1\% Triton X-100, 1\% Nonidet P-40, $25 \mu \mathrm{g} / \mathrm{mL}$ aprotinin, $25 \mu \mathrm{g} / \mathrm{mL}$ leupeptin, $25 \mu \mathrm{g} / \mathrm{mL}$ pepstatin A, and $1 \mathrm{mM}$ phenylmethylsulfonyl fluoride). After centrifugation at $10,000 \mathrm{~g}$ for 15 minutes, the supernatant was used for Western blot analysis. Protein concentration was determined by the Bio-Rad Protein Assay kit (Bio-Rad, Hercules, CA, USA). Aliquots of cell lysates containing $50 \mu$ g of protein were separated by $3 \%$ SDS-polyacrylamide gel electrophoresis and transferred to nitrocellulose membranes (Bio-Rad). Membranes were next incubated with either survivin (sc-10811), Bcl-2 (sc-509), caspase-3 (sc-7148) antibodies followed by appropriate HRP-conjugated secondary antibodies.

Next, the membranes were developed by using an enhanced chemiluminescence detection system (Amersham, Little Chalfont, UK). To confirm equal protein loading, membranes were subsequently reprobed with anti-actin antibody. The reacted individual bands were detected with an image analyzer (LAS-2000, Fuji Film, Tokyo, Japan). All Western blots were performed at least three times for each experiment.

\section{Statistical analysis}

Student t-test was used for comparison of the values between two groups. A P-value of $<0.05$ was designated as the level of significance.

\section{RESULTS}

The effect of artesunate on the growth of HCT116 colon cancer cells

HCT116 colon cancer cells were treated with various concentrations of ART for 24 and 48 hours and the cell viability was assessed by MTT assay. After 48 hours of treatment, the proliferation of HCT116 colon cancer cells was significantly inhibited on 80 and $160 \mu \mathrm{M}$ of the concentration of ART (Fig. 1A). Significant inhibition of cell proliferation also has shown in phase contrast image and Hoechst 33258 staining images (Fig. 1B; a: phase contrast image, b: image of nucleus staining with Hoechst 33258).

Artesunate inhibits the growth of HCT116 colon cancer cells by apoptosis

To determine whether the growth inhibition of ART is related to apoptosis, HCT116 colon cancer cells were treated with ART for 48 hours and analyzed using flow cytometry. As revealed in Fig. $2 \mathrm{~A}$, the proportion of apoptotic cells increased from $1.36 \%$ to $10.27 \%$ on ART treatment. The results indicate that ART may inhibit the growth of HCT116 colon cancer cells by inducing apoptosis.

Next, we determined whether the apoptosis by ART was dependent on ROS or other apoptosis-related proteins. Growth inhibition of HCT116 colon cancer cells by ART does not rescue by co-treatment of ROS scavenger Trolox $(0.5 \mathrm{mM})$ and it indicated that the growth inhibition of ART is not dependent on ROS generation (Fig. 2B). As shown in Fig. 2C, Bcl-2 protein expression
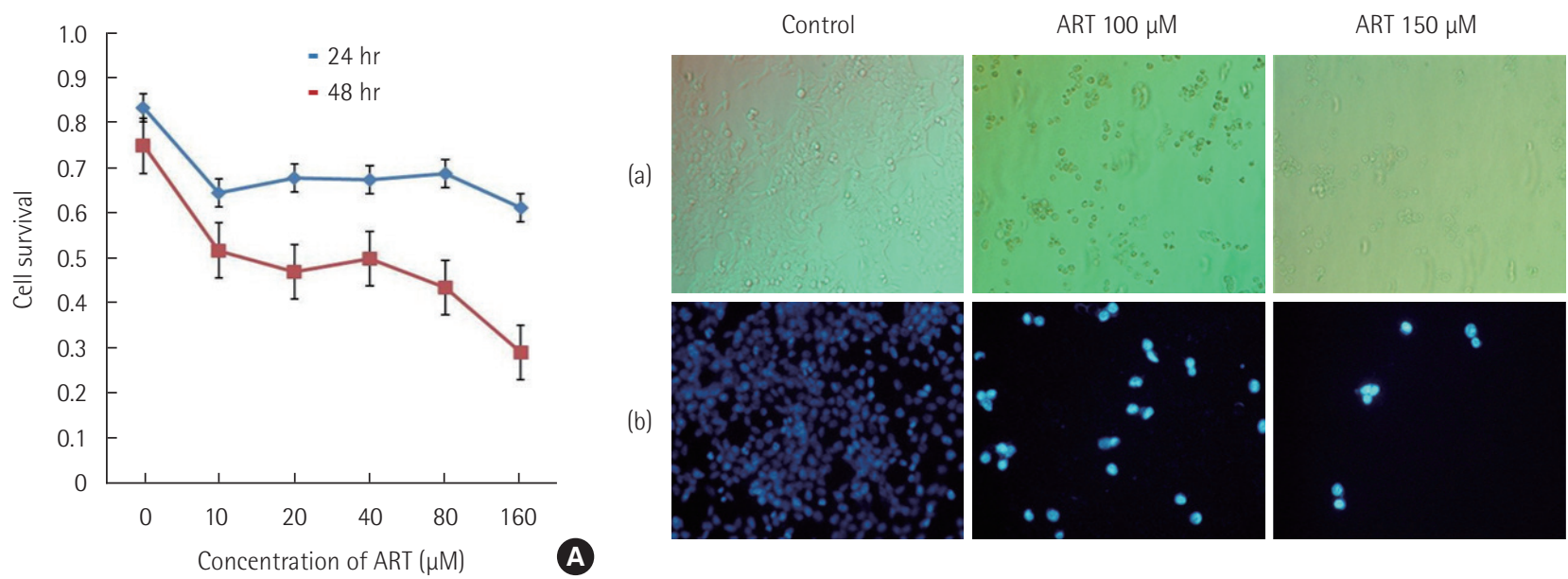

Fig. 1. Artesunate (ART) induces cancer cell death in HCT116 colon cancer cell lines. (A) HCT116 colorectal cancer cells were treated with different concentrations of ART $(0,10,20,40,80$, and $160 \mu \mathrm{M})$ for 24 and 48 hours. Cytotoxicity was assessed using MTT assay. (B) Significant inhibition of cell proliferation has shown in phase on contrast image (a) and nuclear staining by Hoechst 33258 (b). MTT, 3-(4,5-dimethylthiazol-2yl)-2,5-diphenyltetrazolium bromide. 

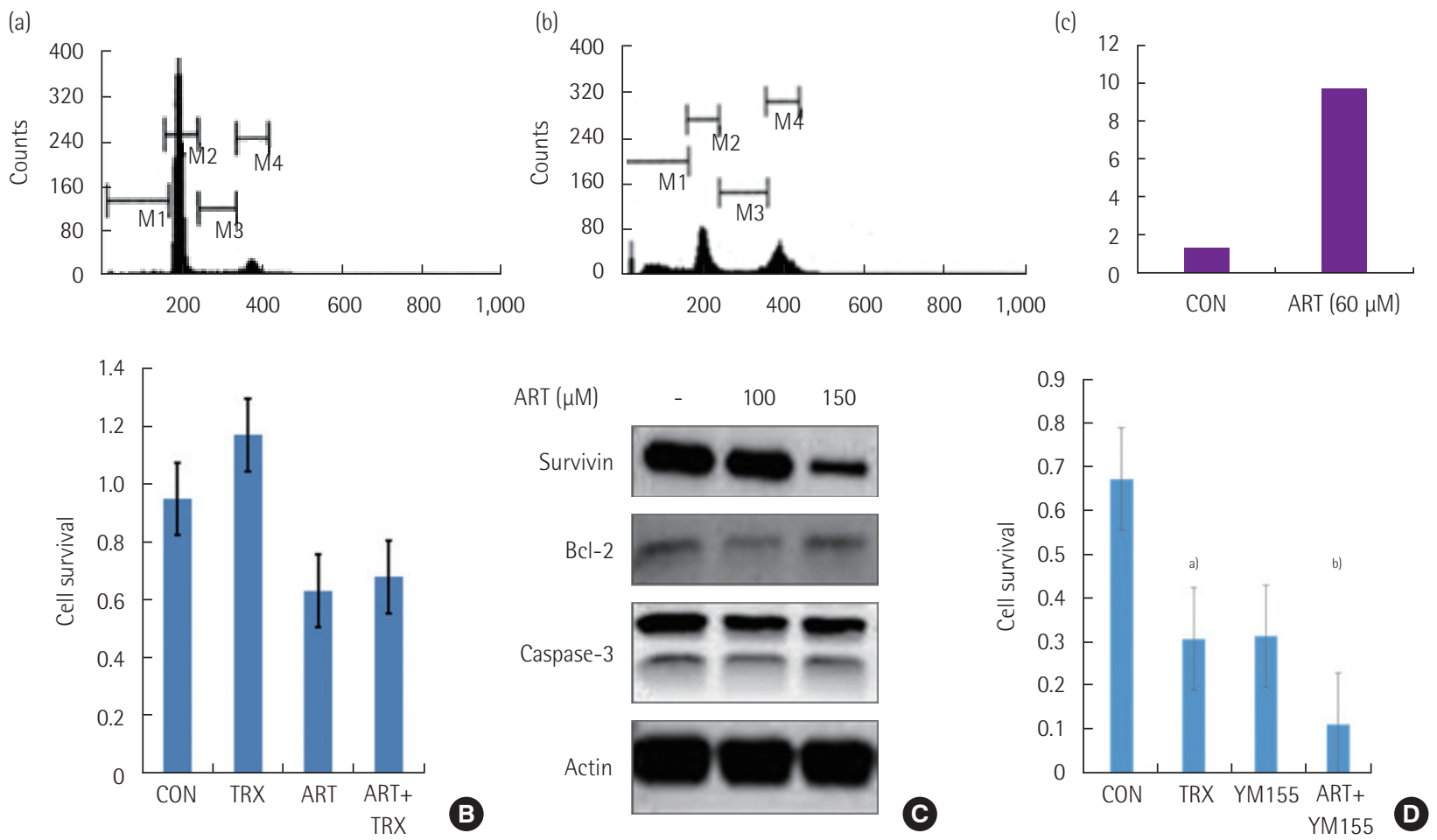

Fig. 2. Artesunate (ART) induces survivin-dependent PCD in HCT116 colon cancer cell lines. (A) HCT116 colon cancer cells were treated with ART $(60 \mu \mathrm{M})$ for 48 hours. Flow cytometric analysis showed that ART induced apoptotic cell death in HCT116 colon cancer cells (a: control, b: treated cells with ART, c: a ratio of the sub-G1 apoptotic cell population between the control and the treated cells with ART). (B) HCT116 colon cancer cells were treated with ART, without and with antioxidant Trolox (0.5 mM). There was no statistically significant difference regardless of co-treatment with Trolox. (C) Survivin expression was suppressed by ART on Western blot analysis. Bcl-2 protein expression did not suppress by ART and caspase-3 activation did not induce by ART treatment. (D) Co-treatment of YM155 (1 mM), specific survivin inhibitor, with ART enhanced the cytotoxic effect of ART $(n=4)$. PCD, programmed cell death; CON, control; TRX, Trolox. ${ }^{\text {al }} P<0.05$. ${ }^{\text {b }} P<0.001$.

does not inhibit on ART treatment and ART does not induce caspase- 3 activation. However, survivin protein expression was inhibited by ART. To determine the importance of survivin in ART-induced apoptosis, we treated the HCT116 colon cancer cells with YM155 (mM), a specific survivin inhibitor. YM155 inhibits the proliferation of HCT116 colon cancer cells but co-treatment of ART with YM155 can enhance the growth-inhibiting activity of ART significantly (Fig. 2D).

\section{Artesunate induces the iron-dependent cell death in colon} cancer cells

ART-medicated cytotoxicity dependent on the presence of iron is termed as ferroptosis, iron-dependent programmed necrosis. This mode of programmed necrosis is inhibited by small-molecule ferrostatin-1. We determined the effect of iron on the ART-induced cell death. The increasing lysosomal free iron by co-treatment with holo-transferrin (HTF, $20 \mu \mathrm{g} / \mathrm{mL}$ ) increases cell death in HCT116 colon cancer cells but is not significant statistically. However, co-treatment of lysosomal iron chelator DFO $(0.1 \mathrm{mM})$ blocked cell death significantly (Fig. 3A and B), demonstrating iron-dependency of ART-induced cell death in HCT116 colon cancer cells. We performed a colony formation assay to determine ART effects on long-term cell survival and proliferation. As described, co-treatment of ART with DFO partially rescued the ART-induced cell death. Co-treatment with necrostatin-1 or ferrostatin does not rescue the ART-induced cytotoxicity (Fig. 3C and D).

\section{DISCUSSION}

ART has been used to treat malaria and is well documented in drug safety in anti-malarial therapy. Previous studies have revealed that ART has anti-proliferative activity in various tumor cells in vitro and in vivo but no adverse effects on normal cells. Also, ART is effective on chemo-resistance cancer cells, which suggests a different mechanism to other chemotherapeutic agents [24].

Artemisinin has effects on many intracellular signal transduc- 


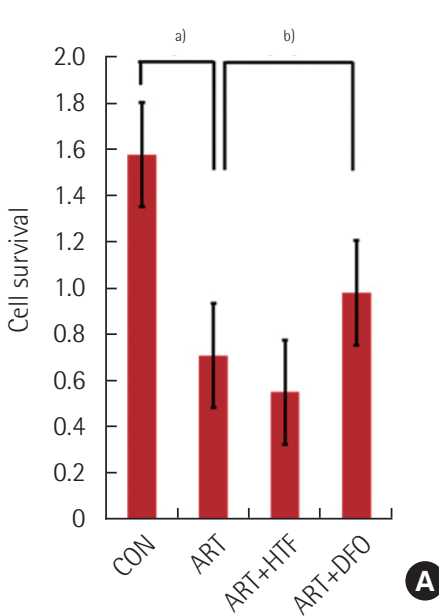

A
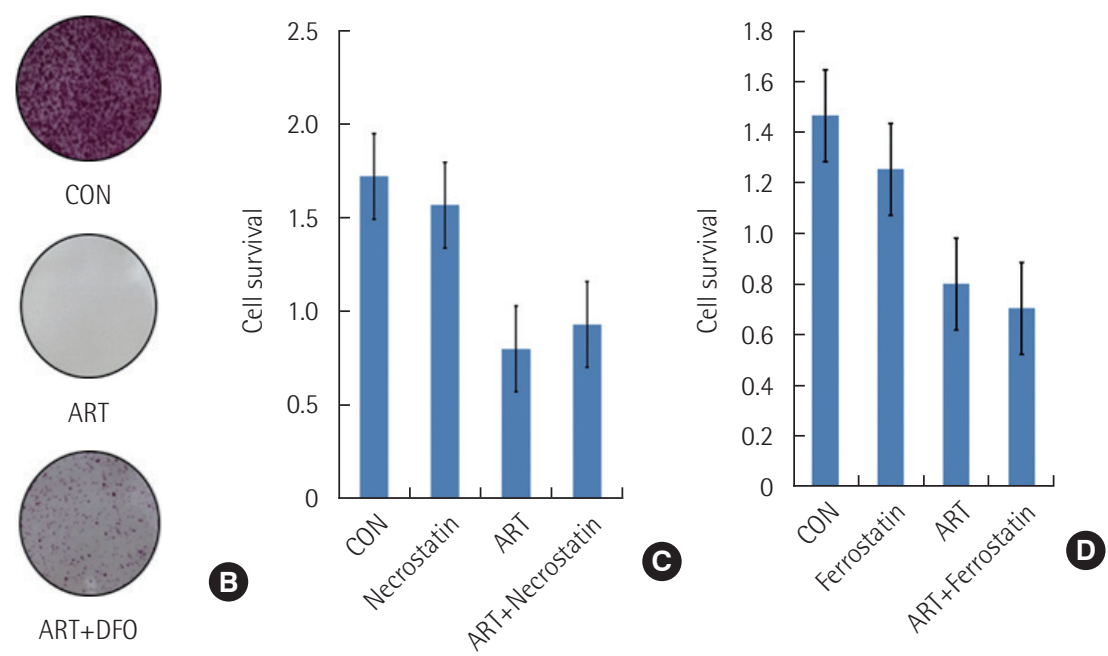

Fig. 3. Artesunate (ART) induces the iron-dependent cell death and does not activate the necroptosis. Addition of holo-transferrin (HTF, 20 $\mu \mathrm{g} / \mathrm{mL}$ ) did not enhance the cytotoxic effect of ART. However, co-treatment with iron chelator deferoxamine (DF0; $0.1 \mathrm{mM})$ reversed the cytotoxic effect of ART $(n=4)(A)$. HCT116 colon cancer cells were subjected to ART and ART+DFO. After 24 hours, 300 surviving cells were re-seeded for a colony-forming assay. After 14 days of re-seeding, the colonies were stained with $1 \%$ (w/v) crystal violet in 25\% methanol (B). The addition of DFO reversed the clonogenic growth inhibition of ART (B). Following colony formation assays were performed and analyzed as in crystal violet staining (B). HCT116 colon cancer cells were subjected to ART with or without the addition of necrostatin-1 (20 $\mu \mathrm{M})$ or ferrostatin $(20 \mu \mathrm{M})$ for 48 hours $(n=3)$. Cell proliferation was assessed using the MT assay, but necrostatin- 1 and ferrostatin could not reverse the cytotoxic effect of ART (C, D). CON, control; MTT, 3-(4,5-dimethylthiazol-2yl)-2,5-diphenyltetrazolium bromide. ${ }^{\text {a) }} \mathrm{P} \leq 0.05$. ${ }^{b)} \mathrm{P} \leq 0.001$.

tions, and it has effects on cellular development, proliferation, and apoptosis. Suppressing the nuclear factor-kB and vascular endothelial growth factor activity is a major mechanism of ART in cancer development [25]. Apoptotic effects on ART are activation of caspase- 3 , caspase-9, and enhancing BAX protein and suppress the Bcl-2 protein $[7,9,11,14]$. ART also induces many molecular substances that activate apoptosis including survivin [26]. Recently, it has been shown that ART-medicated cytotoxicity is dependent on increased ROS generation, the presence of iron, and activates different modes of programmed cell death, including apoptosis, necroptosis, and ferroptosis [10,20].

The mechanism of ART-induced cell death in CRC is not well understood, although it has been proposed that drug-induced cell death occurs in an apoptotic pathway.

In our study, ART induces apoptotic cell death in HCT116 colon cancer cells in the flow-cytometry analysis. Contrary to previous studies, $\mathrm{Bcl}-2$ protein suppression and caspase- 3 activation was not induced with ART treatment in our study. However, survivin protein expression was suppressed with ART treatment. With co-treatment of ROS scavenger, Trolox, ART-medicated cytotoxicity was not affected. Yet, ART-induced cell death is further potentiated by co-treatment with YM155, the specific inhibitor of survivin. These data suggest that the mechanism of the ART-induced cytotoxicity in HCT116 colon cancer cells is dependent on apoptosis by suppressing the survivin expression (Fig. 2). A high level of survivin is related to poor prognosis and resistance to chemotherapeutic agents [22]. ART co-treatment with YM155 can be a new modality to suppress survivin expression for the sensitization of cancer cells to chemotherapeutic agents in colon cancers.

The cytotoxic effect of ART was partially enhanced by transferrin and recovered by chelating ferritin by DFO. Long-term survival was partially restored with co-treatment of ART with DFO. Necroptosis inhibitor does not rescue the cytotoxicity of ART. Therefore, we suggest that the necroptosis pathway does not involve the cytotoxicity of ART in HCT116 colon cancer cells. Even though the cytotoxicity of ART is not enhanced by co-treatment with ferroptosis inhibitor, we strongly suggest that ferroptosis is one of the possible cytotoxic mechanisms of ART in HCT116 colon cancer cells (Fig. 3). In HCT116 colon cancer cells, the mechanism of cytotoxicity of ART may be different from other cancer cells and may be dependent on cancer cell type, the concentration of ART, and exposure time.

A recent small randomized double-blind placebo-controlled trial suggests that ART is the most promising anti-metastatic agent in the post-surgical setting for colorectal cancer. After 42 months following surgery, there was one recurrence in an ART recipient (of 10 patients), and six recurrences in the placebo group (of $12 \mathrm{pa}$ tients). The survival beyond 2 years in the ART group was estimat- 
ed at $91 \%$ whilst only $57 \%$ in the placebo group. This suggests a promising effect of ART on reducing the multiplication of tumor cells in colorectal cancer patients who were already planning to have their cancer surgically removed [16].

Many cell death mechanisms are involved in ART cytotoxicity in different cancer cells, and it differs according to cancer cell types.

We suggest that the mechanism of cytotoxicity of ART in HCT116 colon cancer cells mainly depends on the apoptotic pathway, especially by suppressing survivin expression. Intracellular iron also partially attributes the ART cytotoxicity.

New insight in ART treatment with YM155 will be an effective treatment modality for CRC.

\section{CONFLICT OF INTEREST}

No potential conflict of interest relevant to this article was reported.

\section{REFERENCES}

1. Jung KW, Won YJ, Oh CM, Kong HJ, Lee DH, Lee KH, et al. Cancer statistics in Korea: incidence, mortality, survival, and prevalence in 2014. Cancer Res Treat 2017;49:292-305.

2. Higgins CF. Multiple molecular mechanisms for multidrug resistance transporters. Nature 2007;446:749-57.

3. Klayman DL. Qinghaosu (artemisinin): an antimalarial drug from China. Science 1985;228:1049-55.

4. Pandey AV, Tekwani BL, Singh RL, Chauhan VS. Artemisinin, an endoperoxide antimalarial, disrupts the hemoglobin catabolism and heme detoxification systems in malarial parasite. J Biol Chem 1999;274:19383-8.

5. Kwok JC, Richardson DR. The iron metabolism of neoplastic cells: alterations that facilitate proliferation? Crit Rev Oncol Hematol 2002;42:65-78.

6. Zhang P, Luo HS, Li M, Tan SY. Artesunate inhibits the growth and induces apoptosis of human gastric cancer cells by downregulating COX-2. Onco Targets Ther 2015;8:845-54.

7. Jiao Y, Ge CM, Meng QH, Cao JP, Tong J, Fan SJ. Dihydroartemisinin is an inhibitor of ovarian cancer cell growth. Acta Pharmacol Sin 2007;28:1045-56.

8. Youns M, Efferth T, Reichling J, Fellenberg K, Bauer A, Hoheisel JD. Gene expression profiling identifies novel key players involved in the cytotoxic effect of artesunate on pancreatic cancer cells. Biochem Pharmacol 2009;78:273-83.

9. Lu YY, Chen TS, Qu JL, Pan WL, Sun L, Wei XB. Dihydroartemisinin (DHA) induces caspase-3-dependent apoptosis in human lung adenocarcinoma ASTC-a-1 cells. J Biomed Sci 2009;16:16.
10. Button RW, Lin F, Ercolano E, Vincent JH, Hu B, Hanemann CO, et al. Artesunate induces necrotic cell death in schwannoma cells. Cell Death Dis 2014;5:e1466.

11. Chen H, Sun B, Pan S, Jiang H, Sun X. Dihydroartemisinin inhibits growth of pancreatic cancer cells in vitro and in vivo. Anticancer Drugs 2009;20:131-40.

12. Berger TG, Dieckmann D, Efferth T, Schultz ES, Funk JO, Baur A, et al. Artesunate in the treatment of metastatic uveal melanoma: first experiences. Oncol Rep 2005;14:1599-603.

13. Dell'Eva R, Pfeffer U, Vene R, Anfosso L, Forlani A, Albini A, et al. Inhibition of angiogenesis in vivo and growth of Kaposi's sarcoma xenograft tumors by the anti-malarial artesunate. Biochem Pharmacol 2004;68:2359-66.

14. Hou J, Wang D, Zhang R, Wang H. Experimental therapy of hepatoma with artemisinin and its derivatives: in vitro and in vivo activity, chemosensitization, and mechanisms of action. Clin Cancer Res 2008;14:5519-30.

15. Jansen FH, Adoubi I, J C KC, DE Cnodder T, Jansen N, Tschulakow A, et al. First study of oral Artenimol-R in advanced cervical cancer: clinical benefit, tolerability and tumor markers. Anticancer Res 2011;31:4417-22.

16. Krishna S, Ganapathi S, Ster IC, Saeed ME, Cowan M, Finlayson C, et al. A randomised, double blind, placebo-controlled pilot study of oral artesunate therapy for colorectal cancer. EBioMedicine 2014;2:82-90.

17. Singh NP, Verma KB. Case report of a laryngeal squamous cell carcinoma treated with artesunate. Arch Oncol 2002;10:279-80.

18. Dixon SJ, Lemberg KM, Lamprecht MR, Skouta R, Zaitsev EM, Gleason CE, et al. Ferroptosis: an iron-dependent form of nonapoptotic cell death. Cell 2012;149:1060-72.

19. Eling N, Reuter L, Hazin J, Hamacher-Brady A, Brady NR. Identification of artesunate as a specific activator of ferroptosis in pancreatic cancer cells. Oncoscience 2015;2:517-32.

20. Christofferson DE, Yuan J. Necroptosis as an alternative form of programmed cell death. Curr Opin Cell Biol 2010;22:263-8.

21. Mita AC, Mita MM, Nawrocki ST, Giles FJ. Survivin: key regulator of mitosis and apoptosis and novel target for cancer therapeutics. Clin Cancer Res 2008;14:5000-5.

22. Yamamoto H, Ngan CY, Monden M. Cancer cells survive with survivin. Cancer Sci 2008;99:1709-14.

23. Altieri DC. Validating survivin as a cancer therapeutic target. Nat Rev Cancer 2003;3:46-54.

24. Efferth T, Sauerbrey A, Olbrich A, Gebhart E, Rauch P, Weber HO, et al. Molecular modes of action of artesunate in tumor cell lines. Mol Pharmacol 2003;64:382-94.

25. Lai HC, Singh NP, Sasaki T. Development of artemisinin compounds for cancer treatment. Invest New Drugs 2013;31:230-46. 
Eui Tae Kim et al. • YM155 enhances artesunate-induced cytotoxicity

26. Mu D, Chen W, Yu B, Zhang C, Zhang Y, Qi H. Calcium and survivin are involved in the induction of apoptosis by dihydroartemis- inin in human lung cancer SPC-A-1 cells. Methods Find Exp Clin Pharmacol 2007;29:33-8. 